Revue des patrimoines

17 | 2011

Les patrimoines de l'enseignement supérieur

\title{
Archives et mémoires étudiantes : « non-lieu » de « mémoire »?
}

Jean-Philippe Legois

\section{OpenEdition}

Journals

Édition électronique

URL : http://journals.openedition.org/insitu/2885

DOI : 10.4000/insitu.2885

ISSN : $1630-7305$

Éditeur

Ministère de la culture

Référence électronique

Jean-Philippe Legois, «Archives et mémoires étudiantes : «non-lieu » de « mémoire » ? », In Situ [En ligne], 17 | 2011, mis en ligne le 01 mars 2012, consulté le 30 avril 2019. URL : http:// journals.openedition.org/insitu/2885; DOI : 10.4000/insitu.2885

Ce document a été généré automatiquement le 30 avril 2019.

\section{(c) $($ i) $(9)$}

In Situ Revues des patrimoines est mis à disposition selon les termes de la licence Creative Commons Attribution - Pas d'Utilisation Commerciale - Pas de Modification 4.0 International. 


\title{
Archives et mémoires étudiantes : "non-lieu » de « mémoire»?
}

\author{
Jean-Philippe Legois
}

1 Les artefacts estudiantins, objets témoins de l'évolution des mondes et mouvements étudiants, font partie intégrante du patrimoine mobilier des universités. Plusieurs exemples de patrimonialisation des mondes et mouvements étudiants existent à l'étranger.

2 Mais, dans le cas français, il en est de ce patrimoine mobilier comme des autres types de patrimoines universitaires (immobilier, archivistique, voire immatériel): c'est un patrimoine qui a encore trop peu droit de cité. Et, comme pour les universités, les archives et le patrimoine des mouvements et mondes étudiants ne sont véritablement mobilisés que dans le cadre de célébrations et commémorations ponctuelles. Les archives étudiantes, dans toutes leurs dimensions (structures/ militants, matériel/ immatériel, archives/ documentation/ objets, papier/ électronique), sont donc, pour l'instant, dans une situation tellement marginale qu'elles semblent encore être un «non-lieu» de mémoire.

3 Nous inscrirons cette contribution dans le sillage de la "pertinence de l'impertinence " prônée par le directeur des Archives de l'Université libre de Bruxelles, Didier Devriese. Euvrant depuis plus de 10 ans à la reconnaissance patrimoniale des traces des mouvements étudiants, au sein du GERME (Groupe d'études et de recherche sur les mouvements étudiants) ${ }^{2}$ et de la Mission CAARME $^{3}$, puis de la Cité des mémoires étudiantes, nous plaiderons pourtant ici coupables. Car c'est bien la faute d'archivistes, de chercheurs, de documentaristes et de bien d'autres amateurs éclairés si l'aspect lacunaire, «en miettes $»^{4}$, dispersé, éclaté, «éparpillé par petits bouts, façon puzzle $»^{5}$ de ce patrimoine étudiant est si... éclatant !

(fig. $n^{\circ} 1$ ) 


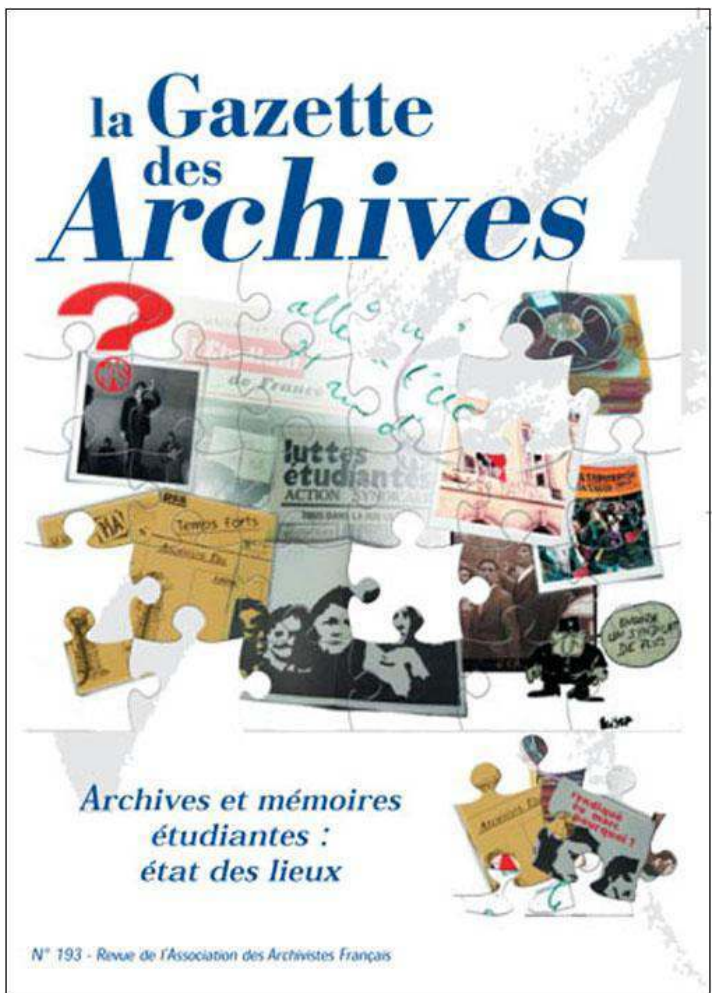

Couverture du numéro spécial de « La Gazette des Archives » consacré aux « archives et mémoires étudiantes ».

(c) Cité des mémoires étudiantes, fonds documentaire, 2004

5 Après avoir établi les charges et les différentes manifestations de décomposition permanente du patrimoine étudiant, puis en avoir recherché les causes (et éventuels coupables) et prononcé le «non-lieu », nous inverserons totalement l'angle d'attaque et verrons quelles solutions nous avons essayées et nous essayons actuellement pour en sauvegarder et valoriser, malgré tout, des fragments.

\section{À la recherche d'un patrimoine étudiant perdu}

6 Ce cliché est symbolique, voire idéal-typique, du mouvement étudiant contemporain, de la force qu'il souhaitait et pouvait dégager, et de la place que les élites étaient prêtes à lui accorder... Et donc du patrimoine potentiel qu'il pouvait produire et transmettre.

7 (fig. $\mathbf{n}^{\circ}$ ) 
Figure 2

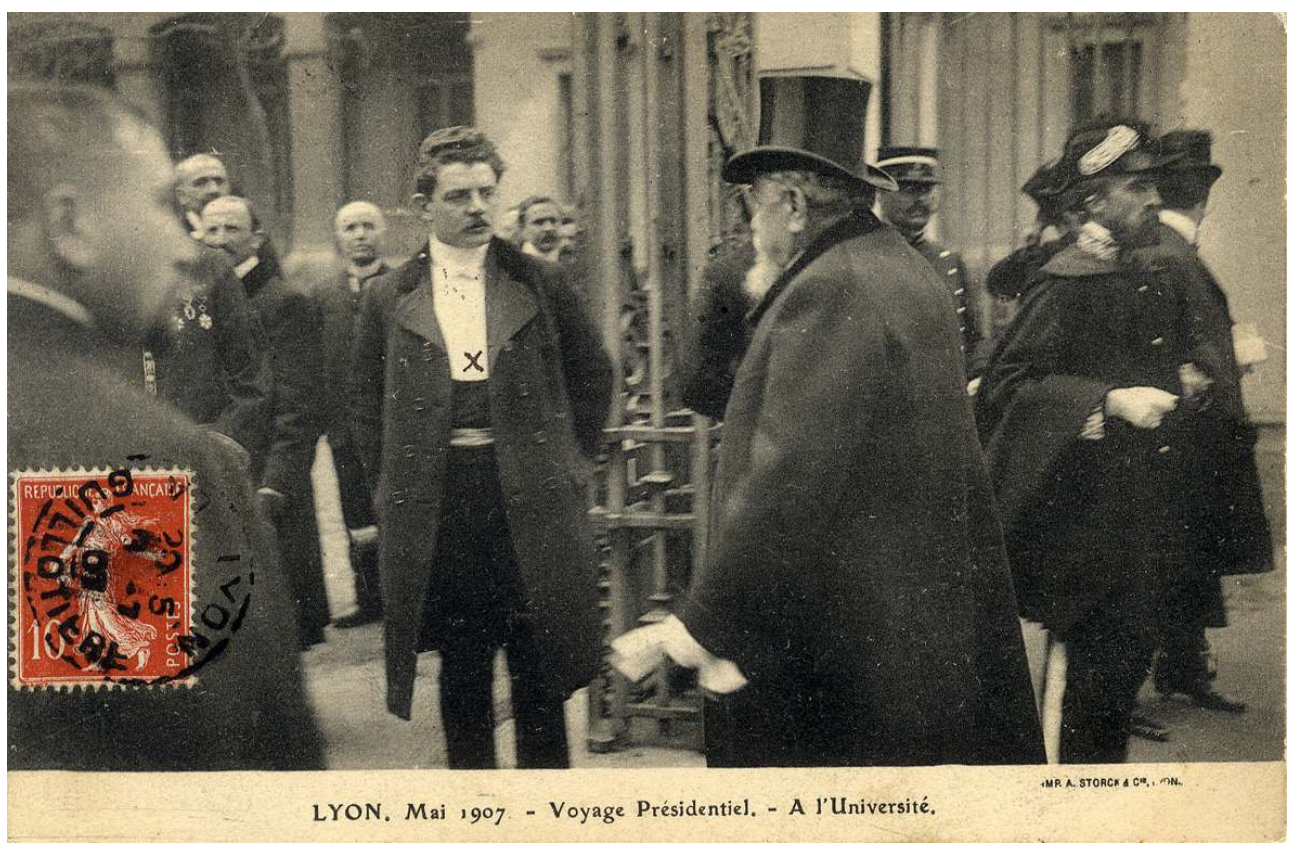

Édouard Herriot, Maire de Lyon, accueillant le président de la République à l'université.

(C) Cité des mémoires étudiantes, fonds documentaire, 1Fi105, 1907.

\section{Splendeurs disparues}

8 On y voit, en 1907, Édouard Herriot, tout jeune maire de Lyon depuis 2 ans, accueillir pour sa visite à l'université de Lyon le président de la République d'alors, Armand Fallières. Ce pourrait être le symbole des jeunes élites, sorties de l'université, choyées par celles déjà en place. Il se trouve qu'Édouard Herriot, maire de Lyon jusqu'en 1957 (avec une interruption de 1942 à 1945) sera plus tard président du Conseil en 1924, 1926, 1932. En outre, en 1935, alors qu'il est ministre d'État, il inaugure le premier sanatorium des étudiants de France à Saint-Hilaire du Touvet, près de Grenoble. C'est alors la première réalisation nationale de l'Union nationale des associations étudiantes de France regroupant depuis 1907 les Associations générales des étudiants (AGE) : même si l'État a finalement apporté des moyens financiers indispensables, les étudiants conservent une présence non-négligeable au Conseil d'administration; l'Union nationale a sa «montagne magique $»^{6}$.

(fig. $n^{\circ} 3$ ) 


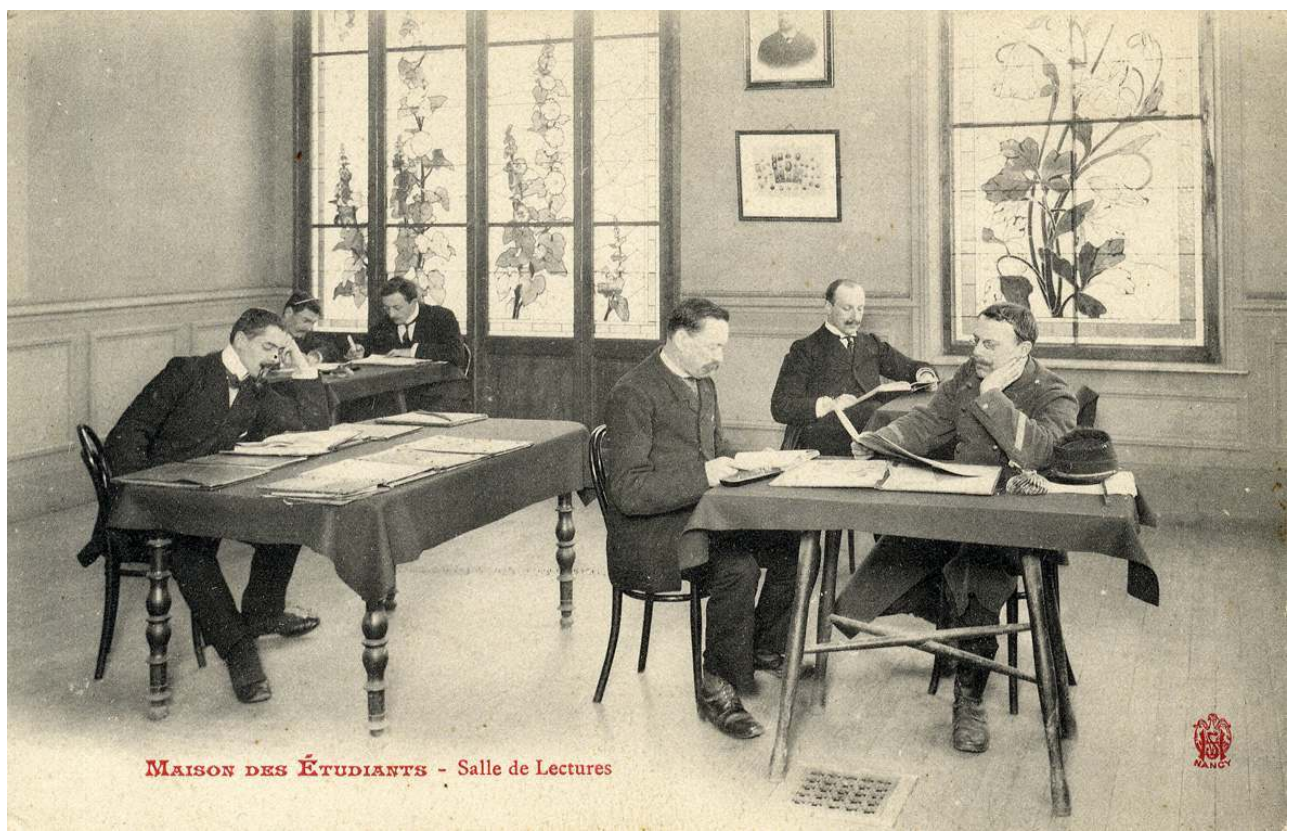

Maison des étudiants de Nancy, fin XIX ${ }^{\top}$ siècle.

(c) Cité des mémoires étudiantes, fonds documentaire, 1 Fi84.

Et il est vrai que, dans chacune des quinze villes universitaires que compte la troisième République conquérante, la Maison des étudiants, siège de l'AGE, n'est pas un lieu insignifiant. La première de ces AGE, créée en 1877 à Nancy, a pour siège social ce qui est aujourd'hui le Musée des Beaux-Arts de la capitale lorraine: le 1, rue Gustave Simon abritera le siège de l'AGEN (une coopérative et un restaurant universitaire) jusqu'en... 1985. L'AGE de Montpellier, après plusieurs changements, fixe son siège, en 1934, dans l'hôtel des 3-5 rue de la Croix-d'or. Celle de Paris a ses locaux au 43, rue des Écoles, puis déménage, en 1910 au 15, rue de la Bûcherie dans un immeuble comprenant grande salle de réunion, salles d'études, bureaux, salle d'escrime, bibliothèque. Cet immeuble est en outre celui qui abrita, du XV $\mathrm{Xu}^{\mathrm{e}} \mathrm{XVIII}{ }^{\mathrm{e}}$ siècle la faculté de Médecine de l'Université de Paris. Quant à celle de Lille, qui accueille, en 1907, le congrès fondateur de l'Union nationale, elle a son siège au 49 , rue de Valmy et y organise restaurant universitaire, imprimerie, coopérative et... salle de billard.

11 Mais l'exemple le plus éclatant, puisque toujours d'actualité, c'est celui de l'Association fédérative générale des étudiants de Strasbourg (AFGES) : constituée à la réintégration de l'Alsace au territoire national français, cette AGE fédérative devient locataire à titre gracieux de locaux appartenant à l'université; en janvier 1927, avec l'autorisation du recteur Charléty, l'AFGES rachète même le bail commercial de l'ancienne brasserie Schutzenberger, "Taverne de l'université ", qui devient le restaurant universitaire «Gallia », ouvrant ses portes le 2 février 1927. L'originalité strasbourgeoise est que ce restaurant est toujours géré actuellement par l'AFGES, suite à une des ironies de l'histoire : à cause ou "grâce " aux militants pro-situationnistes - qui en ont pris la majorité en 1966 -, l'AFGES a été mise sous tutelle judiciaire et a donc échappé aux vicissitudes de l'après mai 1968 et notamment à la nouvelle scission de l'UNEF en $1971^{7}$. 
Figure 4

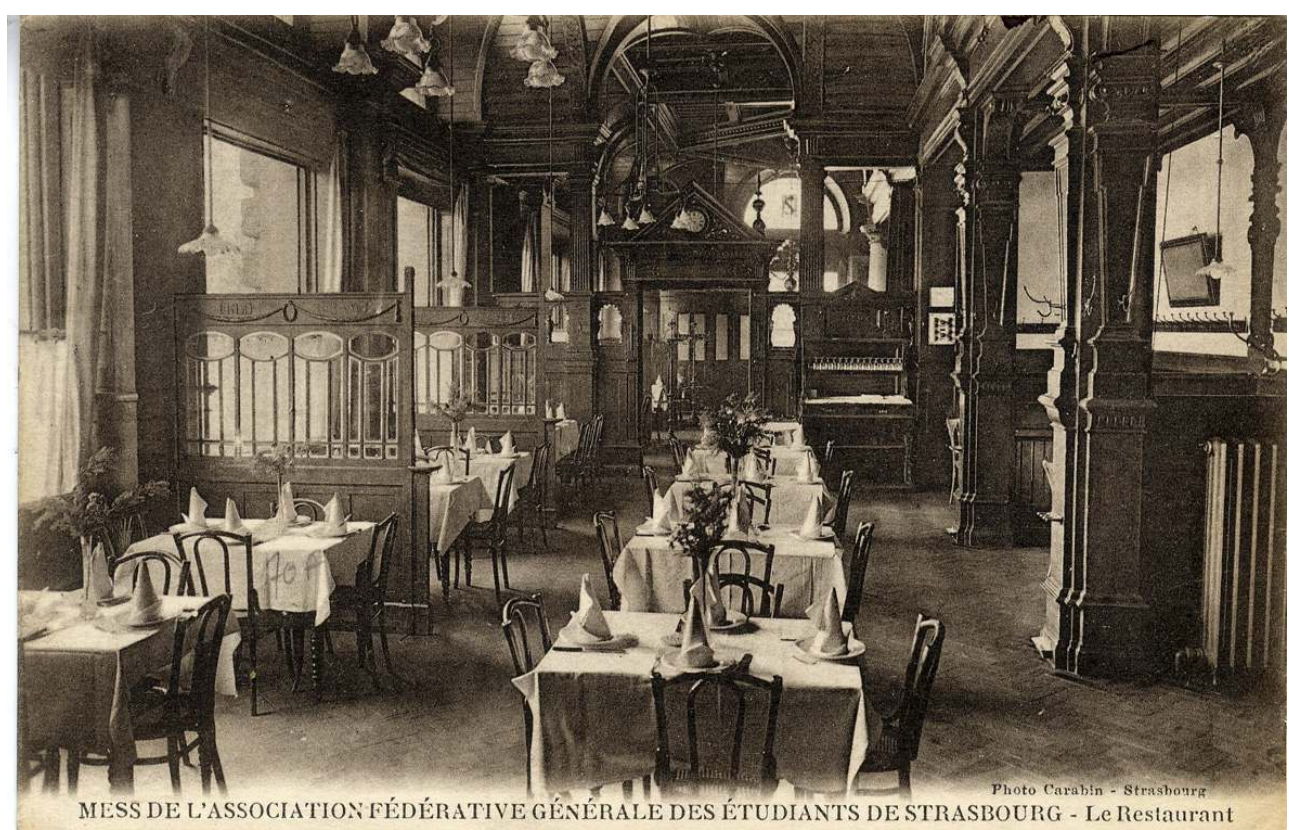

Mess du restaurant « Galia » de l'AFGES, Strasbourg, années 1920.

(c) Cité des mémoires étudiantes, fonds documentaire, 1 Fi87.

13 L'exception strasbourgeoise confirme donc la règle: dans les années 1970, voire parfois 1980, tout ce qui reste de patrimoine immobilier des AGE disparaît. 
Figure 5

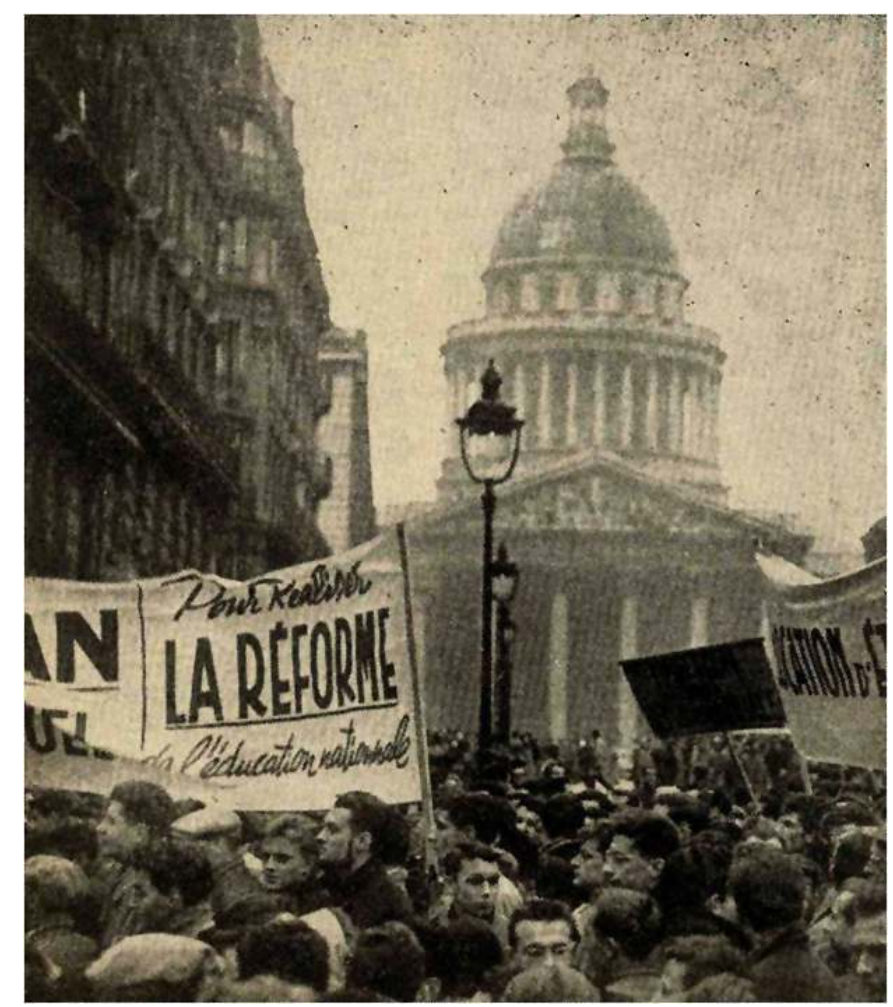

Photographie d'une manifestation, rue Soufflot devant le siège de l'UNEF, extraite de « L'étudiant de France ».

(c) Cité des mémoires étudiantes, fonds documentaire, 1959.

Sur le plan nationaliónion nation cogestion étudiante a été une réalité, non seulement à la Fondation sanatorium des étudiants de France (FSEF), mais aussi au Centre national (et aux Centres régionaux) des œuvres universitaires et scolaires, cette cogestion ayant été inscrite dans la loi du 16 avril 1955 ; la gestion directe étudiante du régime spécifique de sécurité étudiante a même été accordée par la loi du 23 septembre 1948. participation, au CNOUS, à la FSEF ... et dans les nouveaux conseils de gestion des universités créés par la loi Faure après le mouvement de mai-juin 1968. À partir de juin 1960, suite à son engagement contre la guerre d'Algérie, l'UNEF est privée de subvention du gouvernement. Le personnel salarié du syndicat étudiant se réduit... ainsi que la précision et la volumétrie des documents de congrès ${ }^{8}$. Si une des tendances de l'UNEF (Unité syndicale) conserve bien le siège national de la rue Soufflot, après la scission de 1971, cette situation ne durera que quelques années, l'UNEF-US devant déménager pour la rue de Hanovre dès 1976. Ce sont du reste plus encore les scissions que les déménagements (l'une entraînant souvent l'autre) qui sonnent souvent le glas (ou le temps du sauvetage) pour une bonne partie des archives étudiantes.

Il est à noter qu'alors que le nombre d'étudiants en France ne cesse de croître, celui des adhérents à l'UNEF, restée encore unique ou quasiment, va régulièrement se réduire dans 
les années 1960 : entre 1959-60, l'UNEF étant encore une, et 1969-70, juste avant sa nouvelle scission, le nombre d'étudiants passe de 194763 à $625551^{9}$ et le nombre d'adhérents à l'UNEF passe environ de 100000 à $18000^{10}$. L'un est multiplié par plus de 3 , alors que l'autre est... divisé par plus de 5, l'UNEF voyant son taux de syndicalisation passer d'un peu plus de $50 \%$ à même pas $3 \%$. Cet affaiblissement structurel du mouvement étudiant, puis des mouvements étudiants ne peut pas ne pas avoir d'effets sur les patrimoines de ceux-ci.

Le patrimoine immobilier - et autre - des AGE comme de l'Union nationale va donc dépérir et si « la grandeur de ce qui existe donne le regret de ce qui a disparu ${ }^{11}$, le regret est alors immense.

\section{Commémorations ... « Pièges à cons »?}

19 Les commémorations et autres célébrations (nationales ou non) peuvent être des moments, voire des opportunités de renforcement d'un patrimoine, même éclaté et amoindri. Deux conditions, au moins, sont nécessaires pour qu'un tel enrichissement soit possible : une mobilisation des ressources patrimoniales et un appui sur une institution forte dotée d'un organe patrimonial pouvant capitaliser cette mobilisation. Les différentes célébrations qui ont eu lieu ces dernières décennies en France au sein des institutions universitaires n'ont pas réuni ce minimum, contrairement à d'autres configurations nationales (comme, par exemple, l'université de Lausanne, qui crée son service d'archives à l'occasion de son $450^{\mathrm{e}}$ anniversaire en 1987).

Pour ce qui est des archives et mémoires étudiantes en France, les commémorations et autres anniversaires de l'histoire étudiante pourraient constituer une sorte de « seconde chance ", permettant, au-delà de toute utilisation de la mémoire dans une stratégie de constitution, reconstitution ou renforcement d'une identité collective, la structuration lente et patiente d'un véritable patrimoine documentaire ou mobilier. En plus des apports à cette éventuelle capitalisation patrimoniale, ce pourrait être un laboratoire expérimental d'une véritable sociologie de la mémoire, où la commémoration est intimement liée à son ou ses publics, et notamment au secteur de la société dont il est censé renforcer l'identité12 ; laboratoire d'autant plus intéressant que le "public" étudiant est en perpétuel renouvellement.

Le mouvement étudiant a déjà compris et expérimenté l'intérêt des commémorations, cependant, non seulement ces "opérations" sont ponctuelles, mais encore elles sont menées, trop souvent, dans une logique instrumentale de pure communication. Certaines de ses structures, encore fortes, ont pu et su saisir l'occasion de certains de leurs anniversaires pour célébrer leur identité et leur histoire à la lumière des enjeux du moment. À commencer par l'UNEF, dès 1957, qui s'« invente » alors une «tradition " $^{13}$ : alors qu'elle est en train de connaître une scission sur la question de la guerre d'Algérie, elle va célébrer en grandes pompes son cinquantième anniversaire, non seulement en organisant les Jeux universitaires (du 31 août au 5 septembre 1957) et le Festival culturel international (du 9 au 15 septembre 1957) ${ }^{14}$, mais surtout en "commandant » - lors du congrès qui voit pourtant 17 AGE quitter l'UNEF - un livre sur son histoire à deux de ses responsables nationaux, François Borella et Michel de La Fournière. Le syndicalisme étudian $t^{15}$ devient «la bible du syndicalisme étudiant en France ${ }^{16}$, " historique et justification de l'orientation du syndicalisme étudiant depuis $1946 »^{17}$, date de la charte de Grenoble définissant l'étudiant comme un «jeune travailleur intellectuel». (fig. $\mathbf{n}^{\circ} \mathbf{6}$ ) 
Comme l'écrit Paul Bouchet à propos de ce « mythe » grenoblois de l'étudiant, il existe des «mythes utiles et valables ${ }^{18}$, voire « mobilisateurs " ainsi que l'avait déjà écrit Georges Sorel à propos de la grève générale ${ }^{19}$ pour la CGT syndicaliste révolutionnaire du début du $\mathrm{XX}^{\mathrm{e}}$ siècle.

Figure 6

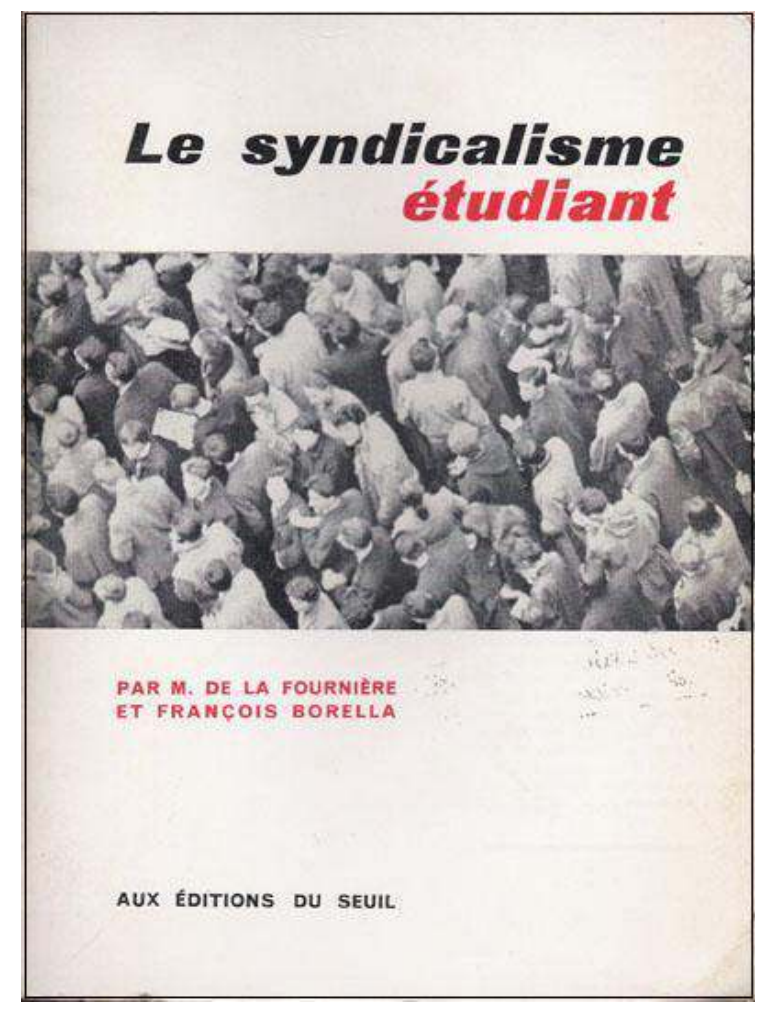

Couverture du livre de Michel de La Fournière et François Borella « Le syndicalisme étudiant » paru aux éditions du Seuil.

(c) Cité des mémoires étudiantes, fonds documentaire, 1957.

En 1988, la Mutuelle nationale des étudiants de France (MNEF) fête ses 40 ans. Elle édite également un ouvrage, retraçant ses grandes périodes et exposant plusieurs «belles pièces » de son patrimoine archivistique... actuellement toujours entre les mains de son liquidateur judiciaire. Dans un contexte de développement de mutuelles régionales concurrentes, d'une certaine marchandisation de la santé et d'une certaine dérive technocratique, l'appel à l'esprit des origines semblait nécessaire.

D'autres anniversaires ont eu lieu plus récemment, mais dans un contexte d'éclatement et d'affaiblissement du mouvement étudiant. La mission de préfiguration (ex-Mission CAARME) de la Cité des mémoires étudiantes ayant été lancée dans une logique parfois "substitutionniste» (voir infra), elle a pu prendre l'initiative de créer un cadre permettant d'organiser des événements célébrant ces grandes dates, mais sans parvenir à compenser, même provisoirement, la fragmentation du mouvement étudiant. 
Figure 7

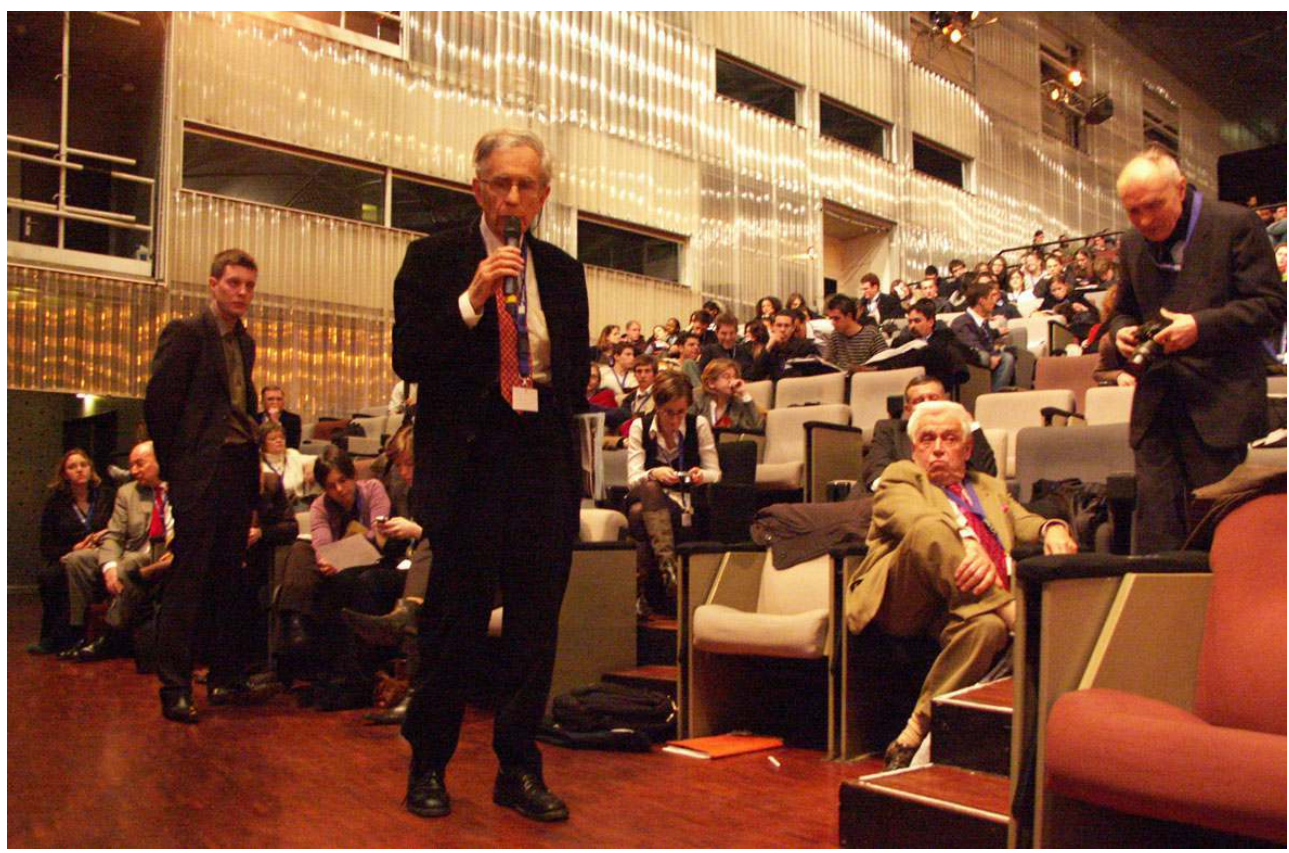

Pierre-Yves Cossé, président de I'UNEF lors de son cinquantenaire, prenant la parole au congrès de I'UNEF en mars 2007.

Phot. Chalier, Caroline. (C) Cité des mémoires étudiantes, 2007

(fig. $\mathbf{n}^{\circ} 7$ )

Dès 2006, la mission organise, avec le musée de la Résistance et de la Déportation de l'Isère, un débat pour les soixante ans de la Charte de Grenoble aux Archives départementales de l'Isère et, en 2007, elle contribue à inscrire le centenaire de la création de l'Union nationale étudiante aux célébrations nationales, mais l'implication des mouvements étudiants à vocation représentative est faible, si ce n'est que tel ou tel s'investit pleinement pour célébrer son histoire dans cette histoire commune ${ }^{20}$. En 2008, à l'occasion des quarante ans de mai 1968, nous avons changé notre fusil d'épaule et avons décidé de prendre les devants en organisant, avec le GERME, dès les 25 et 26 janvier, le colloque "À la redécouverte des mouvements étudiants dans les années 68 ", où étaient présents des représentants de l'UNEF, la FAGE, l'UNI et SUD-étudiant, mais cela n'a évidemment pas empêché certaines de ces organisations de faire des plaidoyers prodomo... Attitude qui montre bien les limites, voire les pièges, des commémorations.

Un contre-exemple a été fourni avec la FSEF, grâce aux expériences menées à l'occasion des soixante ans de la mutualité étudiante, en 2008 également. Souhaitant marquer la fermeture du premier sanatorium étudiant de France à Saint-Hilaire du Touvet (près de Grenoble), la FSEF a décidé en 2009 et 2010 d'entamer un travail de mémoire avec la Cité des mémoires étudiantes. Dans cette mission d'accompagnement patrimonial, nous avons $\mathrm{pu}$ les aider, non seulement à réaliser un ouvrage ${ }^{21}$ et une exposition, mais encore à classer leurs archives et à recueillir un certain nombre de témoignages : pour une fois, la commémoration a été conjuguée avec une véritable action patrimoniale, mais la FSEF est une structure atypique dans ce paysage éclaté...

\section{(fig. $\left.n^{\circ} 8\right)$}




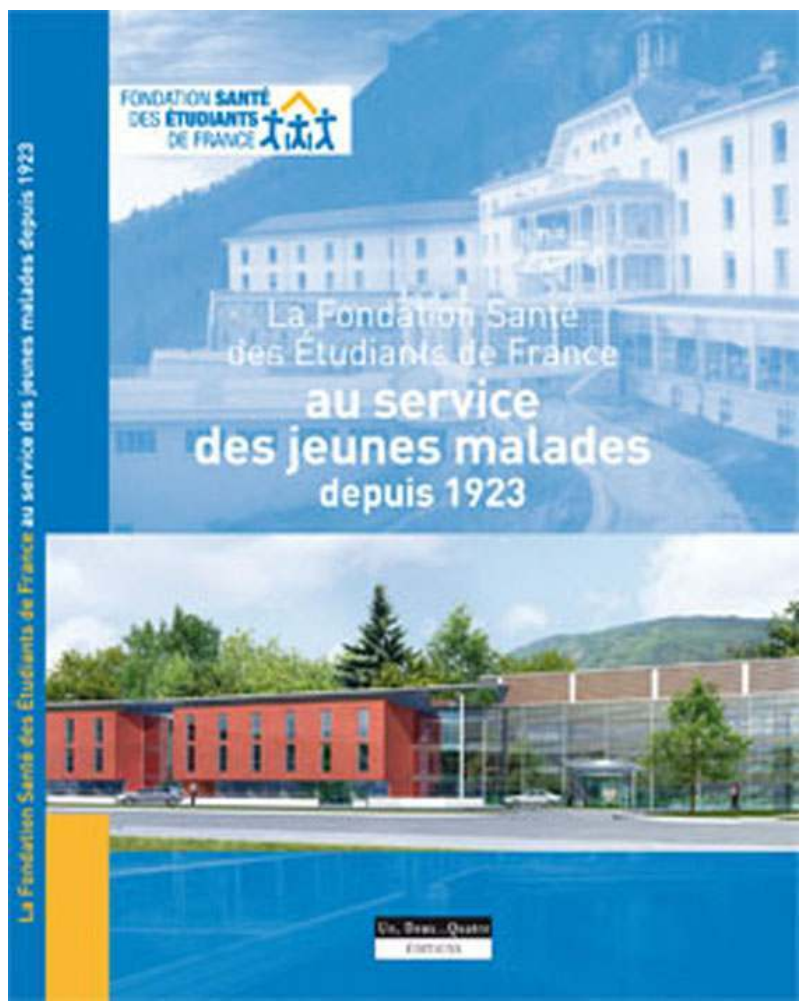

Couverture de l'ouvrage « La Fondation santé des étudiants de France, au service des jeunes malades depuis 1923 ».

(c) Cité des mémoires étudiantes, fonds documentaire, 2010

\section{Un no man's land ... où la guerre des mémoires étudiantes n'aura même pas lieu !}

Un réel patrimoine étudiant est donc difficilement pensable aujourd'hui, mais ses dépouilles pourraient être l'objet de combats entre mouvements étudiants actuels... Or combien de divisions ? À défaut de cathédrales, il existe bien des querelles de chapelles, voire de clochers... Certaines prises de guerre, certains «trophées " peuvent "fortifier " une organisation contre une autre, mais ces guerres ou querelles apparaissent bien comme picrocholines.

S'il y a eu un gâteau à se partager, il n'en reste que des miettes! Et ce no man's land ne sépare que des lignes de belligérants bien clairsemées... Les archives et mémoires étudiantes sont bien un « non-lieu » de « mémoire »!

\section{Vers un « non-lieu » dans « l'affaire » des mémoires étudiantes !}

Pour que cette absence (ou présence fragmentaire) de patrimoine étudiant aboutisse véritablement à un "non-lieu», il nous faut chercher "à qui profite le crime», les éventuels coupables et les disculper. 


\section{« Pouvoir étudiant »... Potere povero!}

31 Premier accusé donc: le mouvement étudiant et ses militant(e)s, producteur(s) de ces archives étudiantes.

32 Si certains partent de l'étymologie grecque du mot archive (arkhe/ commandement) ${ }^{22}$, tous, en tout cas, reconnaissent que la production et - encore plus - la conservation d'archives par un service ad hoc est le propre d'une institution, d'un pouvoir, si petit(e) soit-elle ou soit-il.

33 Aussi les mouvements étudiants apparaissent comme parmi les institutions les plus faibles, si tant est que cette qualification d'institution puisse leur être appliquée.

$34 \mathrm{Au}$ vu de leur tendance lourde et permanente à la décomposition, les mouvements étudiants et leurs archives semblent organiser ce que l'on pourrait appeler un « Fréteval permanent ». Rappelons qu'en 1194, le roi Philippe Auguste y perd une bataille et ses archives qui le suivaient dans ses déplacements, ce qui le décide à sédentariser ses archives dans la capitale du royaume et jusqu'alors donne naissance au «Trésor des chartes ». Le mouvement étudiant, puis les mouvements étudiants, eux, connaissent maintes «catastrophes» documentaires (scissions, déménagements, changements d'orientation), mais n'ont pas la capacité de "sédentariser» leurs archives... Contrairement, par exemple, à la plupart des syndicats de salariés ${ }^{23}$.

Figure 9

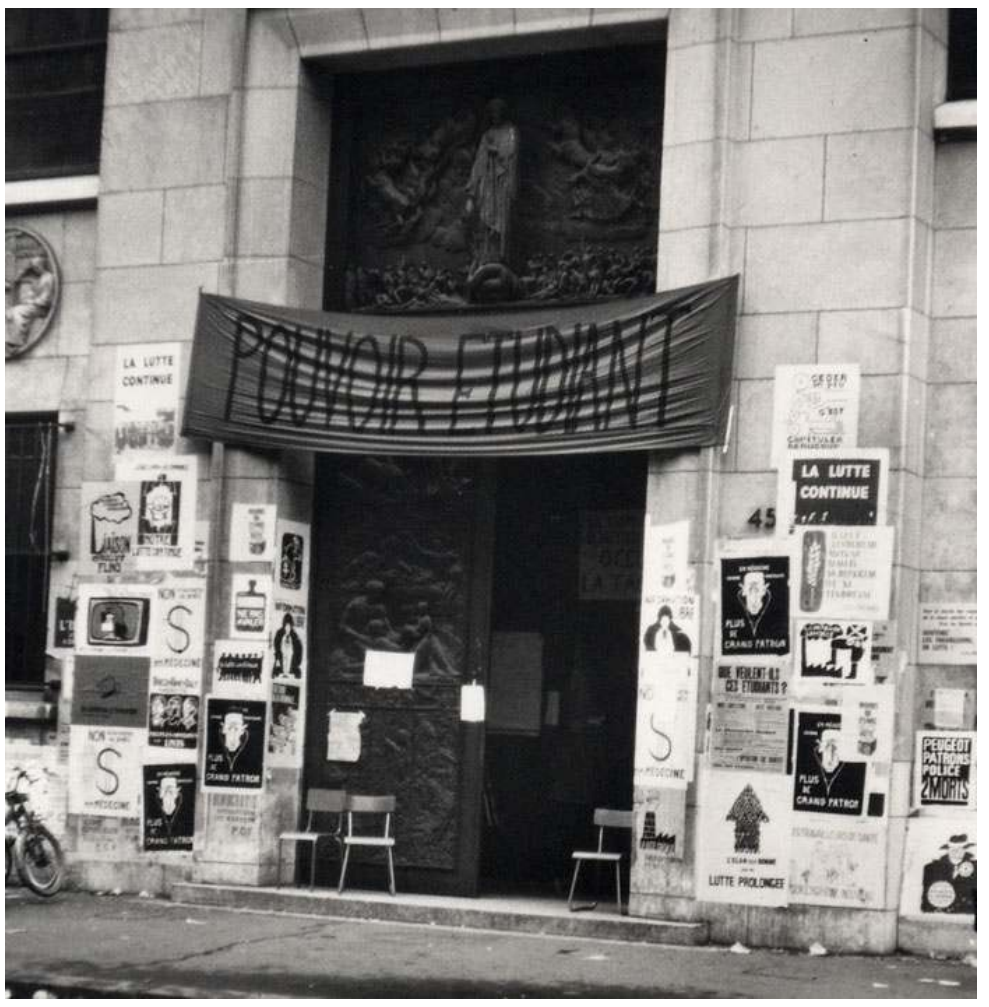

Détail d'une photographie de l'entrée de la « nouvelle » faculté de médecine, mai-juin 1968.

(c) Cité des mémoires étudiantes, fonds documentaire, 7 Fi36.

(fig. $\left.n^{\circ} 9\right)$ 

Outre la relative faiblesse de tout mouvement social, les structures étudiantes cumulent d'autres handicaps : renouvellement rapide des équipes dirigeantes, divisions internes. Une telle faiblesse est à l'origine du caractère fragmentaire du patrimoine documentaire étudiant : le mouvement étudiant peut donc plaider non-coupable !

\section{Des institutions « d'accueil »?}

Caiblesse intrinsèque des archives étudiantes pourrait, en effet, être compensée par l'hospitalité d'institutions fortes, patrimoniales ou de recherche. Comme pour d'autres pouvoirs « déchus» (grandes familles aristocratiques, grandes entreprises familiales ou, dans une certaine mesure, des partis tels le Mouvement républicain populaire, le Parti socialiste unifié ou, encore, les mouvances maoïstes), on aurait pu rêver que les Archives nationales, des Archives départementales ou des institutions patrimoniales telles que la BDIC (Bibliothèque de documentation internationale contemporaine) accueillent non seulement les archives étudiantes de dimension nationale, mais encore un pôle de valorisation.

Comme pour bien d'autres archipels archivistiques, les institutions patrimoniales assurent souvent le rôle de «familles d'accueil $»^{24}$, mais avec une relative parcimonie, en exerçant consciemment ou non une certaine sélection et en ne pouvant pas forcément soutenir une politique de valorisation.

À titre de contre-exemple, citons les Archives départementales de Seine-Saint-Denis, qui ont, non seulement, accueilli en dépôt les archives de la Confédération générale du travail (CGT) ou du Parti communiste français (PCF), mais participent aussi aux actions des structures de valorisation issues de ces mouvements (Institut d'histoire sociale, d'un côté, Archives du communisme, de l'autre).

Pour autant, ne noircissons pas le tableau plus que de raison: comme pour d'autres mouvements de jeunes (la Jeunesse ouvrière chrétienne aux Archives départementales des Hauts-de-Seine, par exemple), il existe de nombreuses institutions tout à fait accueillantes, à commencer par de grandes institutions nationales comme les Archives nationales ou la BDIC ${ }^{25}$. En dehors « des » fonds de l'UNEF (et autres) conservés dans ces établissements, de plus en plus d'Archives municipales ou départementales accueillent de tels fonds : les Archives départementales du Val-de-Marne ont recueilli le fonds de l'AGE Paris 12 ; les Archives municipales de Grenoble, comme celles de Dijon, celui de l'AGE de la ville ; les Archives municipales de Lyon ou de Strasbourg ont collecté les documents du mouvement de mai-juin 1968 dans leur commune. Des centres de recherche, bibliothèques municipales ou universitaires ont pu jouer également ce rôle, mais le fait de classer, conserver et communiquer ces fonds est déjà un investissement important pour des services d'archives qui ne font pas des archives étudiantes le cœur du périmètre de leur collecte. Là aussi, on plaidera non-coupable. 


\section{Le patrimoine documentaire étudiant : des archives « doublement » « orphelines »?}

Aussi, si les archives étudiantes ont bien des producteurs, individuels ou collectifs, plus ou moins pérennes, elles sont très souvent «perdues de vue" par ceux-ci, l'« oubli» pouvant être "délibéré $»^{26}$ : l'héritage "mémoriel » n'est effectivement pas la chose la mieux partagée parmi celles et ceux que l'on a qualifié d' « héritiers ${ }^{27}$. C'est en ce sens que l'on peut parler d'archives orphelines, «doublement» orphelines, puisque les familles d'accueil ont elles-mêmes du mal à aider ces « archives » à trouver une « seconde vie ». La sauvegarde de ces archives est donc souvent déconnectée de leur production.

Dans la situation de "vacance du pouvoir» étudiant décrite auparavant, une archivistique est-elle tout de même possible? Une archivistique sans pouvoir est-elle pensable? Ce que nous avons appelé une "anarchivistique $»^{28}$, qui plus est contrainte, est-elle envisageable?

\section{Fragments d'anarchivistique étudiante}

Un dernier accusé était envisageable: nous-mêmes, archivistes, chercheurs, documentaristes et autres amateurs éclairés de ce patrimoine particulier.

Si, dès l'introduction nous disions plaider coupables, c'était pour pousser jusqu'au bout la logique absurde de vouloir faire rentrer le patrimoine étudiant dans un cadre ne pouvant lui correspondre.

Il serait, en effet, totalement aberrant de « s'excuser » d'avoir sauvegardé quelques pièces du puzzle étudiant. À archives non-traditionnelles, archivistique particulière.

Renversons donc notre point de vue, arrêtons de marcher sur la tête et remettons notre problème sur ses pieds, inversons la charge de la preuve : on ne peut regarder les archives étudiantes en chaussant des lunettes institutionnelles. Hors des sentiers battus, voyons quelles archivistiques buissonnières nous avons pu explorer et à partir de quels foyers et maquis nous pouvons reprendre l'offensive.

\section{Les limites d'une anarchivistique participative ou substitutionniste}

L'air rémois a pu, l'espace d'un instant, nous faire croire au mirage de ces « cathédrales élevées à la gloire de la curiosité et du génie humains ${ }^{29}$ que sont les musées et autres institutions patrimoniales. Nous avons pu nous mettre à rêver d'un seul pôle magnétique rassemblant les volets archivistique, documentaire et muséal du patrimoine étudiant. C'était surestimer la capacité des acteurs et amateurs de ce patrimoine à s'unir ainsi que l'impact réel d'un tel patrimoine sur les enjeux bien concrets des collectivités territoriales et des établissements d'enseignement supérieur.

Sans revenir sur certains aspects plus conjoncturels $\mathrm{s}^{30}$, la phase de préfiguration rémoise a permis de cerner les limites d'une anarchivistique tant participative que substitutionniste.

Dans une logique d'éducation populaire et/ou de service public "traditionnel», nous avons pu, un moment, penser à développer une seule structure pouvant embrasser l'ensemble des questions patrimoniales liées aux mouvements étudiants. Nous avons pu 
croire à la mobilisation commune et relativement massive non seulement de collectivités territoriales et d'établissements d'enseignement supérieur, mais aussi de toutes les strates des collectivités territoriales et des ministères (tout au moins de ceux chargés de la culture et de l'enseignement supérieur), mais une telle substitution patrimoniale s'appuie elle-même - nous l'avons vu plus haut - sur une institution déjà établie dont les fées ne se penchent que sur des berceaux leur semblant dignes d'intérêt - au vu des critères du temps présent; nous butons là sur la légitimité encore en construction de ce type de patrimoine et sur celle des mouvements étudiants comme objet de recherche.

51 (fig. $\left.\mathbf{n}^{\circ} \mathbf{1 0}\right)$

Figure 10

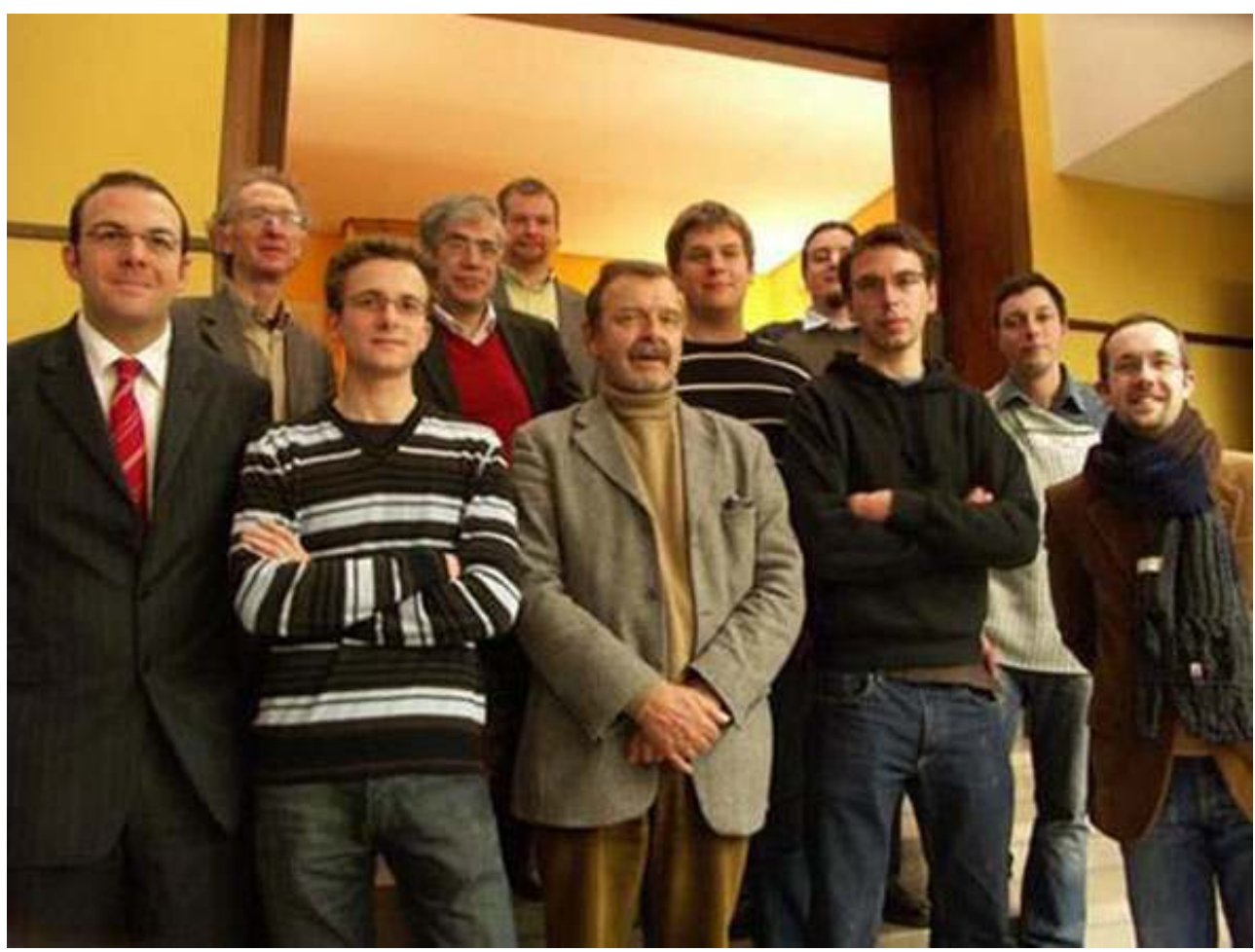

Installation du Comité national consultatif étudiant, le 14 décembre 2007.

Phot. Chalier, Caroline. ( C Cité des mémoires étudiantes, 2007.

Nous avons aussi voulu associer pleinement à notre démarche les mouvements étudiants actuels et les anciens responsables. Dès 2004 et le lancement de notre mission de préfiguration, nous avons tenté de regrouper, autour du réseau du Conservatoire des mémoires étudiantes, anciens responsables des mouvements étudiants, chercheurs et professionnels des archives et de la documentation. Auprès de chacune des organisations étudiantes, nous avons entamé un travail de sensibilisation, allant de la signature de chartes communes à l'archivage audiovisuel en amont de leurs congrès. En décembre 2007, à l'occasion des quatrièmes journées "Archives et mémoires étudiantes ", nous avons installé un Comité national consultatif étudiant pour associer les différents mouvements étudiants à vocation représentative à nos actions de sauvegarde et de valorisation des archives étudiantes : cela a permis de rassembler sur cette cause de l'UNI à SUD-étudiant, en passant par l'UNEF, la FAGE, PDE et la Cé. 
53 Mais ces différentes tentatives, nécessaires, ont montré, pour autant, leurs limites lorsqu'il a fallu mener une mobilisation commune pour la reconnaissance nationale et ministérielle de cette action patrimoniale. Il est difficile en même temps de soutenir et de se réapproprier pleinement une démarche. Et peut-être étions-nous encore dans un schéma trop institutionnel, tout en voulant pour autant nous allier avec tous les amateurs éclairés. Il est alors devenu raisonnable de renforcer une démarche résolue et raisonnée de professionnels du patrimoine en lien avec la communauté scientifique concernée.

\section{Vers une anarchivistique raisonnée et multipolaire}

54 S'il est vrai que "l'archive s'impose comme fragmentaire, éclatée, révisable et si elle s'offre à toutes les synthèses historiques possibles $»^{31}$, nous nous sommes donc proposés de prendre les archives étudiantes pour chantier-école, terrain expérimental d'une archivistique renouvelée, voire régénérée aux sources de l'archivistique contemporaine, c'est-à-dire une archivistique démocratique, "d'en bas ", d'intérêt général, prenant à bras le corps ces archives non-institutionnelles et pourtant partie des «archives de la nation » au sens de la loi de Messidor an II.

En effet, les structures sociales insuffisamment puissantes pour gérer leurs propres archives ou les faire gérer par des institutions n'en sont pas moins des éléments vivaces de la vie sociale et civique du pays.

Aussi avons-nous petit à petit élaboré et mis en œuvre une politique de réseau documentaire thématique, la future Cité des mémoires étudiantes (ex-Mission CAARME) assurant informellement un rôle de tête de réseau. Des programmes de valorisation régionaux ou thématiques intitulés "Mémoires vives étudiantes» ont été initiés, permettant d'associer plusieurs institutions patrimoniales.

Un programme fondateur a été mené en Île-de-France, qui a permis de numériser des périodiques étudiants nationaux ${ }^{32}$ et franciliens, de recueillir et mettre en ligne des témoignages oraux et, dans le cadre d'un partenariat avec les Archives du rectorat de Paris, de réaliser une exposition multimedia sur « Facultés et lycées dans l'académie de Paris, entre résistance et collaboration", valorisant des documents issus des fonds rectoraux. 


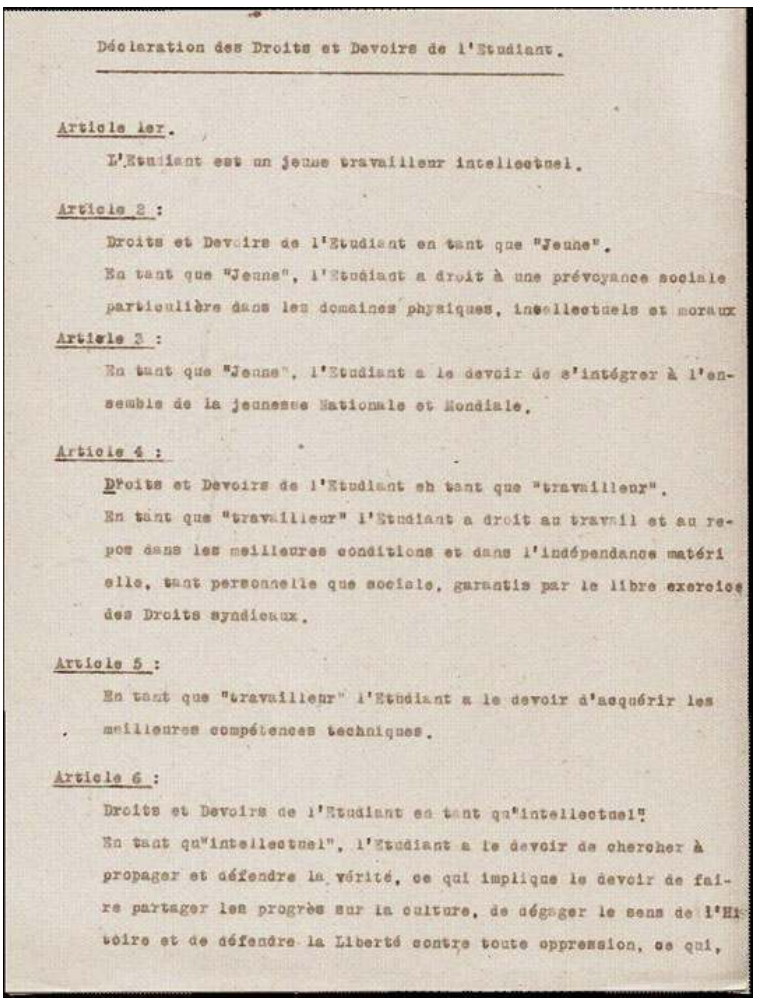

Première page de la Charte de Grenoble, 1946.

(c) Cité des mémoires étudiantes, fonds documentaire.

\section{(fig. $\left.\mathbf{n}^{\circ} 11\right)$}

Le programme «Mémoires vives étudiantes en Rhône-Alpes » a été mené en 2006, à l'occasion des soixante ans de la «Charte de Grenoble », avec le soutien du ministère de la Culture et de la Communication (direction régionale des affaires culturelles de RhôneAlpes) et du Conseil régional de Rhône-Alpes, en partenariat avec le Laboratoire Triangle, les Archives municipales de Grenoble, Lyon, Saint-Étienne et la BDIC. Ce programme a non seulement permis de valoriser et reconstituer numériquement des collections de presse étudiante matériellement et institutionnellement éclatées ${ }^{33}$, mais a permis également de collecter de nouveaux fonds, notamment celui de Jean-Paul Martin, aux Archives de Saint-Étienne, et celui de l'UNEF-ID, aux Archives de Lyon...

Dans le prolongement du soixantième anniversaire de la création du régime étudiant de Sécurité sociale (1948), le programme « Mémoires vives étudiantes : mutualité et santé »a rassemblé, notamment, en 2009-2010, la Fondation santé des étudiants de France (FSEF), la BDIC et la Cité des mémoires étudiantes autour des objectifs de numérisation et mise en ligne de témoignages oraux et de presse étudiante sur ces thèmes, grâce entre autres au soutien du ministère de la Culture et de la Communication (Mission recherche et technologie). Ce programme peut en particulier s'appuyer sur le travail autour des archives de la FSEF.

D'autres exemples pourraient être cités, comme le «Journal électronique de la Commune étudiante " réalisé à l'occasion des quarante ans de mai-juin $1968^{34}$ ou l'exposition multimedia «Panorama de la presse étudiante catholique à travers le monde en 100 vues » et les colloques « Étudiant(e)s en mouvements » tenus en 2006, à l'occasion du 
classement du fonds d'archives de la Jeunesse étudiante catholique internationale (JECI ${ }^{35}$, dans le cadre de la convention BDIC-Cité... Mais, pour qu'une telle politique de réseau thématique fonctionne, il est effectivement important de pouvoir compter sur une tête de réseau solide et souple.

Figure 12

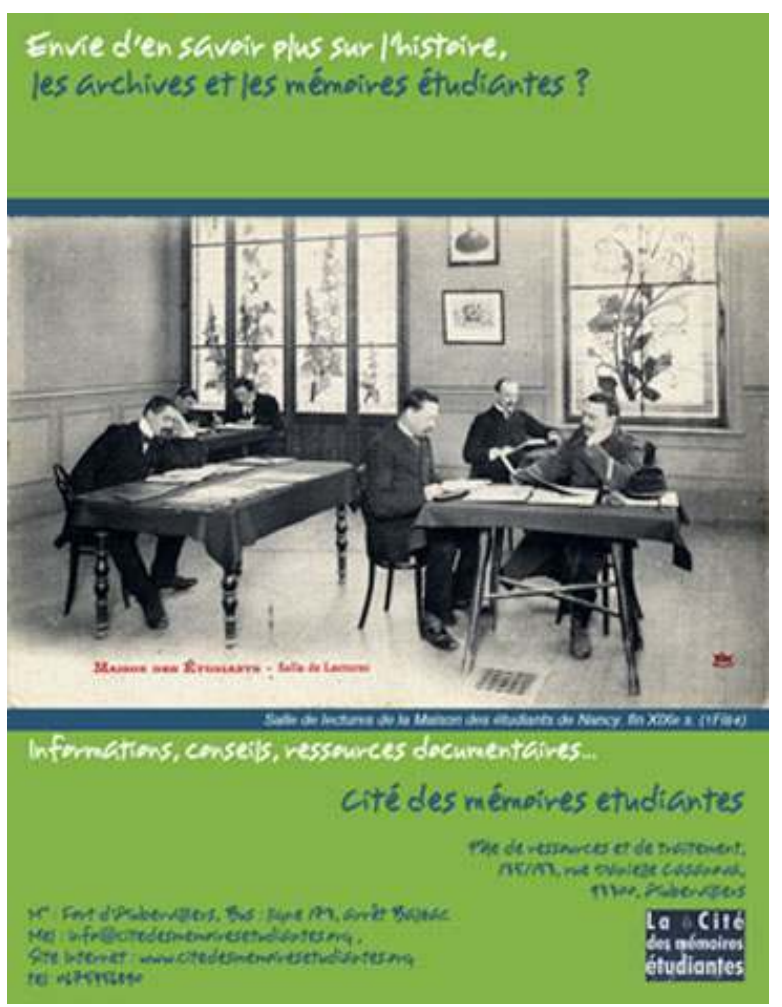

Affiche du nouveau pôle de ressources de la Cité des mémoires étudiantes à Aubervilliers.

(c) Cité des mémoires étudiantes, fonds documentaire, 2011 son pôle de ressources et de traitement à Aubervilliers (Seine-Saint-Denis) dans la perspective du futur développement du campus Condorcet, mais a également "cultivé » un pôle de pré-conservation et de traitement à Beauchamp (Val d'Oise) ${ }^{36}$.

Avec cette démarche multipolaire adoptée lors de notre implantation en région Île-deFrance, nous sommes davantage en capacité d'embrasser les fonds étudiants dans toute leur diversité : fonds de structures comme de militants, mais aussi collectes actives lors de mobilisations.

$\mathrm{Au}$ final, les mouvements étudiants, et donc leur patrimoine, apparaissent comme des organismes en constante évolution, décomposition, recomposition, construction/ déconstruction/ reconstruction : plutôt que de vouloir transposer un schéma patrimonial institutionnel, nul et non-avenu pour ce «non-lieu » de " mémoire », il est plus pertinent d'adopter une stratégie délibérément fragmentaire, où chaque sauvegarde est une petite victoire contre l'oubli.

66 Et, si le mythe de Sisyphe aurait pu être un des mythes constitutifs du mouvement étudiant ${ }^{37}$, il faut effectivement "imaginer Sisyphe heureux », accumulant petit à petit 
quelques bribes de ces « archives du rêve étudiant » pour reprendre la belle formule de Philippe Péchoux... Et de Jacques Rancière ${ }^{38}$.

\section{NOTES}

1. - Didier Devriese a développé cette idée, notamment lors de deux rendez-vous importants pour la prise en compte des archives et mémoires étudiantes: la deuxième journée des Archives organisée les 17 et 18 avril 2002 par le service des archives de l'Université catholique de Louvainla-Neuve (Voir SCHOUKENS, Cathy (Éd). Archives universités, monde étudiant: une mémoire en construction, 2003) et les troisièmes journées « Archives et mémoires étudiantes » tenues à Reims les 14 et 15 octobre 2005.

2. - LEGOIS, Jean-Philippe. "Archives et mémoires étudiantes: enjeu historique et enjeux archivistiques ». Les Cahiers du GERME, $\mathrm{n}^{\circ} 19,2001$, p. 6-14.

3. - En 2003-2004, la ville, puis l'université de Reims proposent au GERME de l'aider dans le développement d'un centre de ressources dédié aux mouvements étudiants, ce qui débouche sur la mission de préfiguration CAARME (2004-2009), pérennisée sous le nom de Cité des mémoires étudiantes... en région Île-de-France.

4. - COHEN, Yolande et WEIL, Claudie (coord). « Entre socialisme et nationalisme, les mouvements étudiants européens ». Le Mouvement Social, $n^{\circ} 120$, juillet-septembre 1982.

5. - AUDIARD, Michel. Les Tontons flingueurs, 1963 (Bernard Blier, alias Raoul Volfoni).

6. - Pour citer Thomas Mann... et Didier Fischer. Voir FISCHER, Didier. Les étudiants en France (1945-1968). Contribution à une histoire socio-culturelle et politique du milieu étudiant. Thèse d'histoire, Université Paris X-Nanterre, 1998 (dir. de Jean-Jacques Becker) ; FISCHER, Didier, MORDER Robi. La Fondation santé des étudiants de France, au service des jeunes maladies depuis 1923. FSEF, Un, deux... quatre éditions, 2010.

7. - Pour plus de précisions sur la situation de ces AGE et d'autres : RAEIS, Olivier. «Les AGE de l'UNEF. Leurs statuts juridiques, essai de recensement. Bref aperçu de 1880 à nos jours ». Dans Les Cahiers du GERME, "Engagements étudiants ", spécial n³, février 1998, p. 65-72 et spécial n4, 2003/2004, p. 35-40.

8. - Voir « Archives et mémoires étudiantes : état des lieux ». La gazette des Archives (coord. JeanPhilippe Legois), n¹93, 2004 (préface de Martine de Boisdeffre). Ce numéro spécial a rassemblé les actes des deux premières journées «Archives et mémoires étudiantes » (BDIC, 2000 ; CPU, 2001).

9. - PASSERON, Jean-Claude. «1950-1980 : l'Université mise à la question ». Dans VERGER, Jacques (dir.). Histoire des Universités en France. Toulouse : Privat, 1986, p. 397.

10. - MONCHABLON, Alain. Histoire de l'UNEF. Paris : PUF, 1983, p. 106 ; LEGOIS, Jean-Philippe, MONCHABLON, Alain, MORDER, Robi. Cent ans de mouvements étudiants. Paris : Syllepse, 2007, p. 98. 11. - À propos de la mosquée de Cordoue: M'RABET, Fadela. Le muezzin aux yeux bleus. Paris : Riveneuve éditions, 2008, p. 21.

12. - GENSBURGER, Sarah et LAVABRE, Marie-Claire. " Un point de vue sur la controverse autour de la «Maison de l'Histoire de France ». La sociologie de la mémoire comme tierce position. » Dans BABELON, Jean-Pierre, BACKOUCHE, Isabelle, DUCLERT, Vincent, JAMES-SARAZIN, Ariane. Quel musée d'histoire pour la France. Paris : Armand Colin, 2011, p. 89. 
13. - ORKIBI, Eithan. L'ethos collectif dans la rhétorique des mouvements sociaux : l'Union nationale des étudiants de France pendant la guerre d'Algérie (1954-1962). Thèse, Université de Tel Aviv, 2011 (dir. de Ruth Amossy), p. 249-257.

14. - « 1907, une union étudiante est née ». Matériaux pour l'histoire de notre temps, n86, avril-juin 2007 ; ORKIBI, Eithan. L'ethos collectif dans la rhétorique des mouvements sociaux : l'Union nationale des étudiants de France pendant la guerre d'Algérie (1954-1962). Thèse, Université de Tel Aviv, 2011 (dir. de Ruth Amossy), p. 216.

15. - BORELLA, François et DE LA FOURNIÈRE, Michel. Le syndicalisme étudiant. Paris : éditions du Seuil, 1957.

16. - MONCHABLON, Alain. « 1946 : le congrès de la charte de Grenoble ». Dans MORDER, Robi (dir.). Naissance d'un syndicalisme étudiant. 1946 : la charte de Grenoble. Paris : Syllepse, 2006, p. 63-70, p. 63.

17. - MONCHABLON, Alain. «Borella François, Charles, Joseph, André ». Les cahiers du GERME, $n^{\circ}$ 22-23-24, décembre 2002.

18. - BOUCHET, Paul. «Conditions et perspectives de l'action étudiante ». Esprit, n ${ }^{\circ}$, avril 1952, p. 588. Cité par ORKIBI, Eithan. L'ethos collectif dans la rhétorique des mouvements sociaux: l'Union nationale des étudiants de France pendant la guerre d'Algérie (1954-1962). Thèse, Université de Tel Aviv, 2011 (dir. de Ruth Amossy), p. 64.

19. - SOREL, Georges. Réflexions sur la violence. Paris : Marcel Rivière et Cie, 1908.

20. - C'est à cette occasion que Jean-Michel Rodrigo lance le projet du documentaire «L'UNEF et les frondes étudiantes » qui n'aboutit qu'en... 2011.

21. - FISCHER, Didier, MORDER Robi. La Fondation santé des étudiants de France, au service des jeunes maladies depuis 1923. FSEF, Un, deux... quatre éditions, 2010.

22. - Heinz Wismann, Colloque « Les Français et leurs archives », 5 novembre 2001.

23. - Regroupés avec les autres centres liés aux mouvements sociaux au sein du CODHOS (Collectif des centres de documentation en histoire ouvrière et sociale): http:// www.codhos.asso.fr

24. - Nous empruntons cette notion à la réflexion de Paul Klimpel, directeur administratif du Museum für Film und Fernsehen de Berlin, sur les œuvres orphelines (comme son "plaidé » « coupable »), voir Le 64e congrès de la Fédération internationale des archives du film (FIAF), 17-26 avril 2008, Cinémathèque française.

25. - Sur l'ensemble des partenariats avec la BDIC concernant les archives et mémoires étudiantes, voir LEGOIS, Jean-Philippe et MORDER, Robi. « De la sauvegarde à la valorisation des archives étudiantes : un exemple de coopération thématique ». Dans Matériaux pour l'histoire de notre temps, $\mathrm{n}^{\circ} 100$, «Pratiques de recherche et collections de la BDIC », octobre-décembre 2010, p. 46-52.

26. - MONCHABLON, Alain. "Le mouvement étudiant et sa mémoire : l'UNEF après 1945, entre tradition et oubli ». Dans L'homme et la société, n¹11-112, Générations et mémoires, janvier-juin 1994, 1-2. Paris : L'Harmattan, p. 113-117, p. 116.

27. - BOURDIEU, Pierre et PASSERON, Jean-Claude. Les héritiers : les étudiants et la culture. Paris : Minuit, 1966.

28. - LEGOIS, Jean-Philippe. Archives et recherches : spécificités, contradictions et convergences (à partir $d u$ cas des archives étudiantes), séminaire du GERME au Centre d'histoire de Sciences-Po, Paris, 16 mars 2009. Une partie de cette intervention se retrouve dans cette contribution.

29. - JAMES-SARAZIN, Ariane. «La création du musée de l'Histoire de France aux Archives nationales au XIXe siècle». Dans BABELON, Jean-Pierre, BACKOUCHE, Isabelle, DUCLERT, Vincent, JAMES-SARAZIN, Ariane. Quel musée d'histoire pour la France? Paris : Armand Colin, 2011, p. 108.

30. - CHALIER, Caroline et LEGOIS, Jean-Philippe. « Où en est la Cité des mémoires étudiantes ? Où vont les archives étudiantes? ». Les cahiers du GERME, n²8, 2009, p. 7-9. 
31. - JAMES-SARAZIN, Ariane. «Quel avenir pour le musée de l'Histoire de France aux Archives nationales?». Dans BABELON, Jean-Pierre, BACKOUCHE, Isabelle, DUCLERT, Vincent, JAMESSARAZIN, Ariane. Quel musée d'histoire pour la France? Paris : Armand Colin, 2011, p. 161.

32. - Outre des titres comme L'étudiant de France, signalons qu'un partenariat spécifique entre la BDIC, la Cité et les Archives du communisme a permis la numérisation et la mise en ligne de nombreux numéros de Clarté.

33. - BOULENC, Anne, CHALIER, Caroline et LEGOIS, Jean-Philippe. « Un exemple de valorisation partagée : la presse syndicale étudiante grenobloise ». Dans La gazette des Archives, Les archives des syndicats et des mouvements sociaux, $\mathrm{n}^{\circ} 221,2011$.

34. - BLUM, Françoise, LEGOIS, Jean-Philippe et MARIN, Anne-Catherine. « Archives, événements et « Commune étudiante » : prolonger et valoriser plusieurs collectes immédiates du mouvement social de mai-juillet 1968 ». Dans La gazette des Archives, Les archives des syndicats et des mouvements sociaux, n²21, 2011.

35. - SUC, Benjamin, LEGOIS, Jean-Philippe. "Fonds de la Jeunesse étudiante catholique internationale ». Matériaux pour l'histoire de notre temps, n86, avril-juin 2007.

36. - Siège également du fonds de dotation «Cité des mémoires étudiantes » permettant de rassembler les dons de particuliers et de structures : 3ter, avenue Hébert, 95250, Beauchamp.

37. - LEGOIS, Jean-Philippe. «Commune étudiante». Dans ARTOUS, Antoine, EPSZTAJN, Didier, SILBERSTEIN, Patrick (dir.). La France des années 1968. Paris : Syllepse, 2008.

38. - Voir PÉCHOUX, Philippe. «Les fonds d'archives collectés en Bourgogne par l'ADIAMOS : les archives du rêve étudiant ». Dans La gazette des Archives (coord. Jean-Philippe Legois), n¹93, 2004 (préface de Martine de Boisdeffre), p. 80.

\section{RÉSUMÉS}

Le patrimoine, notamment archivistique, des mondes et mouvements étudiants, a encore peu droit de cité, particulièrement en France. Après avoir établi différentes disparitions ou fausses apparitions de ce patrimoine en perte constante, nous en rechercherons les causes (éventuels coupables), à commencer par les mouvements étudiants eux-mêmes, sans oublier les professionnels du patrimoine. Puis, après avoir prononcé le non-lieu, nous verrons quelles solutions alternatives ont pu ou peuvent être envisagées pour sauvegarder et valoriser ce qui peut l'être.

Heritage, including archivistical, of the student worlds and movements, has not enough a little legitimate place, particularly in France. After having established various disappearances of this heritage in constant loss, we investigate the causes (possible culprits), starting with the student movements themselves, without forgetting the heritage professionals. Then, after delivering the withdrawal of case, we will see what alternatives have been or may be considered to safeguard and enhance what can be.

INDEX

Mots-clés : étudiant, mouvement étudiant, patrimoine, archives, mémoires, collections, commémoration, sauvegarde, valorisation 
AUTEUR

JEAN-PHILIPPE LEGOIS

Président de la Cité des mémoires étudiantes jplegois@citedesmemoiresetudiantes.org 